\title{
Recent advances in intravital microscopy for investigation of dynamic cellular behavior in vivo
}

\author{
Yeon Woong Choo ${ }^{1}$, Juhee Jeong ${ }^{1}$ \& Keehoon Jung ${ }^{1,2,3, *}$ \\ ${ }^{1}$ Department of Biomedical Sciences, BK21 Plus Biomedical Science Project, Seoul National University College of Medicine, Seoul 03080, \\ ${ }^{2}$ Department of Anatomy and Cell Biology, Seoul National University College of Medicine, Seoul 03080, ${ }^{3}$ Institute of Allergy and Clinical \\ Immunology, Seoul National University Medical Research Center, Seoul 03080, Korea
}

Currently, most biological research relies on conventional experimental techniques that allow only static analyses at certain time points in vitro or ex vivo. However, if one could visualize cellular dynamics in living organisms, that would provide a unique opportunity to study key biological phenomena in vivo. Intravital microscopy (IVM) encompasses diverse optical systems for direct viewing of objects, including biological structures and individual cells in live animals. With the current development of devices and techniques, IVM addresses important questions in various fields of biological and biomedical sciences. In this mini-review, we provide a general introduction to IVM and examples of recent applications in the field of immunology, oncology, and vascular biology. We also introduce an advanced type of IVM, dubbed real-time IVM, equipped with video-rate resonant scanning. Since the realtime IVM can render cellular dynamics with high temporal resolution in vivo, it allows visualization and analysis of rapid biological processes. [BMB Reports 2020; 53(7): 357-366]

\section{INTRODUCTION}

Cells are basic structural and functional units of living organisms. The functions of cells and their interactions with other cells and biological components mediate many crucial biological processes in vivo (1-4). Traditionally, in vitro and/or ex vivo analyses, such as immunohistochemistry, flow cytometry, and cell-based assays, have been used for evaluating location, population, and functions of cells in a given organ of animals. However, these in vitro/ex vivo analyses can obtain information only in the static/dead state of cells, but cannot acquire the data in the moving/live state in a living organism

${ }^{*}$ Corresponding author. Tel: +82-2-740-8207; Fax: +82-2-745-9528; E-mail: keehoon.jung@snu.ac.kr

https://doi.org/10.5483/BMBRep.2020.53.7.069

Received 15 March 2020

Keywords: Cell biology, Cell trafficking, Cellular behavior, In vivo imaging, Intravital microscopy
(5). To overcome these technical obstacles, many researchers have directed efforts toward developing a new device and technique to visualize and investigate biological phenomena in live animals.

One of those efforts is the development of whole-body imaging, such as magnetic resonance imaging (MRI), singlephoton emission computed tomography (SPECT), and positron emission tomography (PET). These techniques can show the whole-body distribution of specific probes, such as fluorescence, radioactive tracers, or contrast enhancements in live animals $(6,7)$. Accordingly, whole-body imaging is currently being used for the diagnosis of various diseases. However, most whole-body imaging has limited resolution (MRI, 10-100 $\mu \mathrm{m}$; SPECT, 1-2 mm; PET, 1-2 mm, in general) that hinders visualizing objects at cellular level (Table 1) (7).

On the other hand, intravital microscopy (IVM) has been developed as an alternative modality that overcomes the limitation of whole-body imaging. IVM, as a microscopic imaging system, has high spatial $(\sim 1 \mu \mathrm{m})$ and temporal resolution (sub-seconds) (8-11). In this regard, IVM has been applied to visualizing and monitoring single-cell biological processes, different from other in vivo imaging modalities (Table 1) $(7,12-18)$. In this mini-review, we will summarize the history of IVM and its applications in terms of investigating cellular behaviors in various fields spanning from vascular biology and immunology to oncology. We will also review recent studies using real-time IVM, showing how this videorate scanning IVM can contribute to the field of cell biology.

\section{Development of IVM - Basic optics of single- and multi-photon microscopy}

IVM incorporates all the microscopy techniques, which can have various resolutions and frame rates, for visualizing and analyzing biological events in living animals. The concept of observing living animals with microscopy was first introduced by the early pioneers of microscopy in the 17th and 18th centuries (19). In the 19th century, Julius Cohnheim used light microscopy to observe the migration of leukocytes in blood vessels toward injury sites in the transparent tongue of a living frog and discovered that their magnetism is a crucial process of inflammation (20). Even though this early conventional optical 


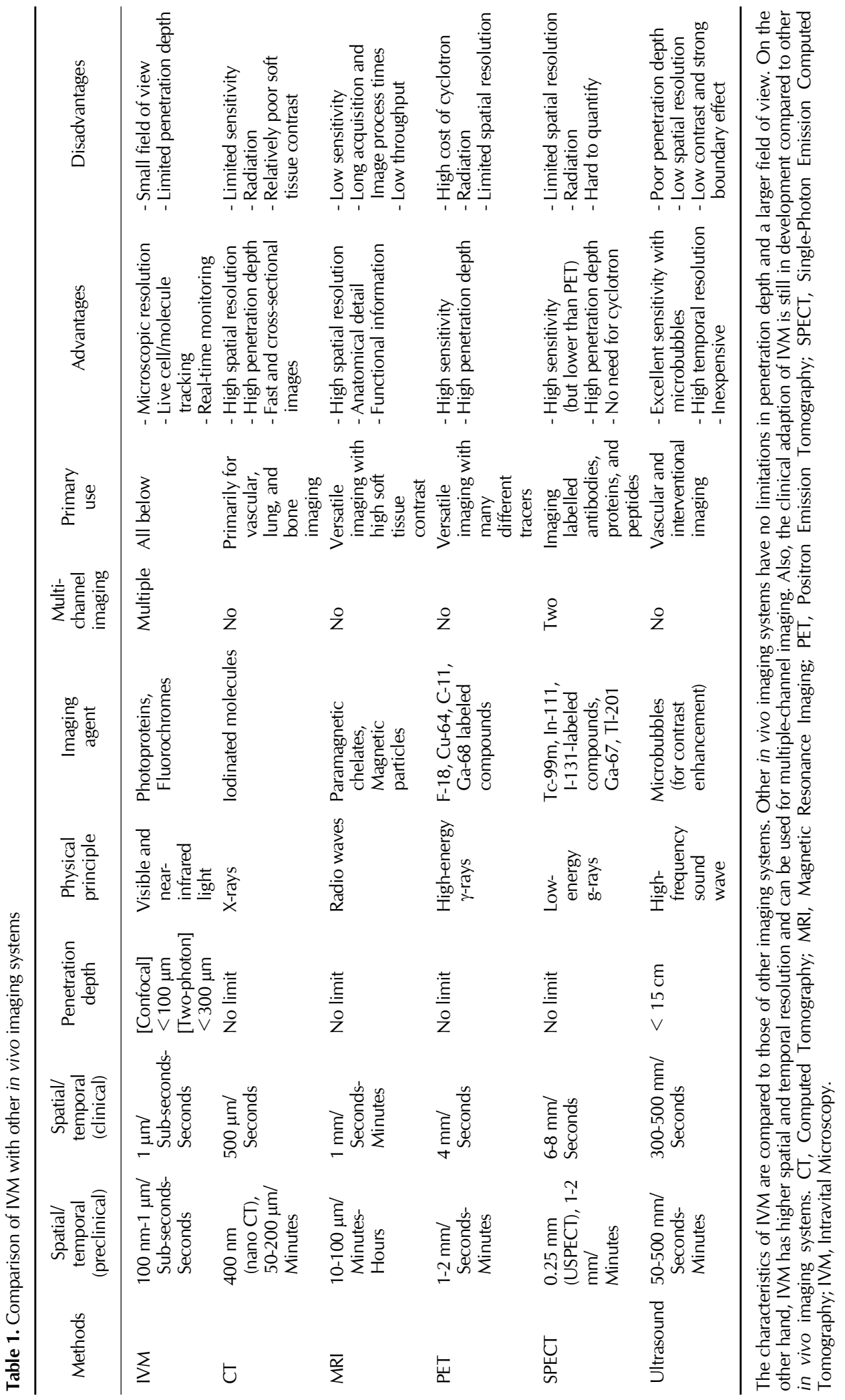


IVM contributed to discovering new aspects of biology, it had many limitations: the difficult reduction of background signals, dependence in the eyesight of the observer, and no controlling depth of field.

After significant development of microscopy and image processing, microscopic imaging techniques, originally built for in vitro imaging, have been adapted to in vivo applications. One of the adapted approaches is confocal microscopy (Fig. 1A). As shown in Fig. 1A, the basic structures of the optics in conventional confocal microscopy and in confocal IVM are similar. In confocal IVM, however, there are additional components required for maintaining steady-state breathing of the animal, which also allows stable imaging. Confocal microscopy uses a point illumination to activate the fluorescence and collects photons emanating from the sample to the detector through the pinhole. The pinhole of confocal microscopy blocks out-of-focus signals, which allows optical sectioning in thick samples. With the development of fluorescent cells and reporter mice, confocal microscopy setups have become widespread for intravital imaging $(19,20)$. However, because of the intrinsic optical properties, the penetration depth of confocal microscopy is only up to $\sim 100 \mu \mathrm{m}$, which limits deep-tissue imaging in living animals. Also, the generally short wavelengths for fluorescence excitation are subject to strong scattering in biological tissues, which can increase phototoxicity in the sample as well (8).

Another adopted approach is multi-photon microscopy (810). The essential mechanism of multi-photon (e.g., two-photon) microscopy is to induce fluorescence in the laser-focused site with the summed energy of the photons in the laser. The basic structures of confocal and two-photon microscopy - most conventional multi-photon microscopy - are compared in Fig. 1B and 1C. In confocal (single-photon) microscopy, the exciting laser shoots a photon that has enough energy to induce the emission of fluorescence, which occurs in all the areas that the laser hits, thus creating the necessity for the pinhole that rejects the signals from out-of-focus areas. In contrast, in twophoton microscopy, the fluorescence is generated in a small region where the laser is tightly focused, since only the sites which two photons excite receive enough energy to emit the fluorescence. As a result, the out-of-focus signal can be rejected in multi-photon microscopy without needing another element like a pinhole. Also, the use of long-wavelength excitation has the advantage in imaging deep tissues because it can minimize scattering and absorption by certain proteins in tissues (11). Thus, the two-photon laser allows a deep tissue penetration of $300 \mu \mathrm{m}$, which is superior to that of confocal imaging (18), and causes negligible photodamage or photobleaching (7). Furthermore, the second-harmonic generation (SHG) can be observed in two-photon microscopy. SHG is a second-order non-linear optical process in which two photons interacting with non-linear optical material (e.g., collagen) combine to form a new photon with twice the frequency and half the wavelength of the initial photons (21). With these
A

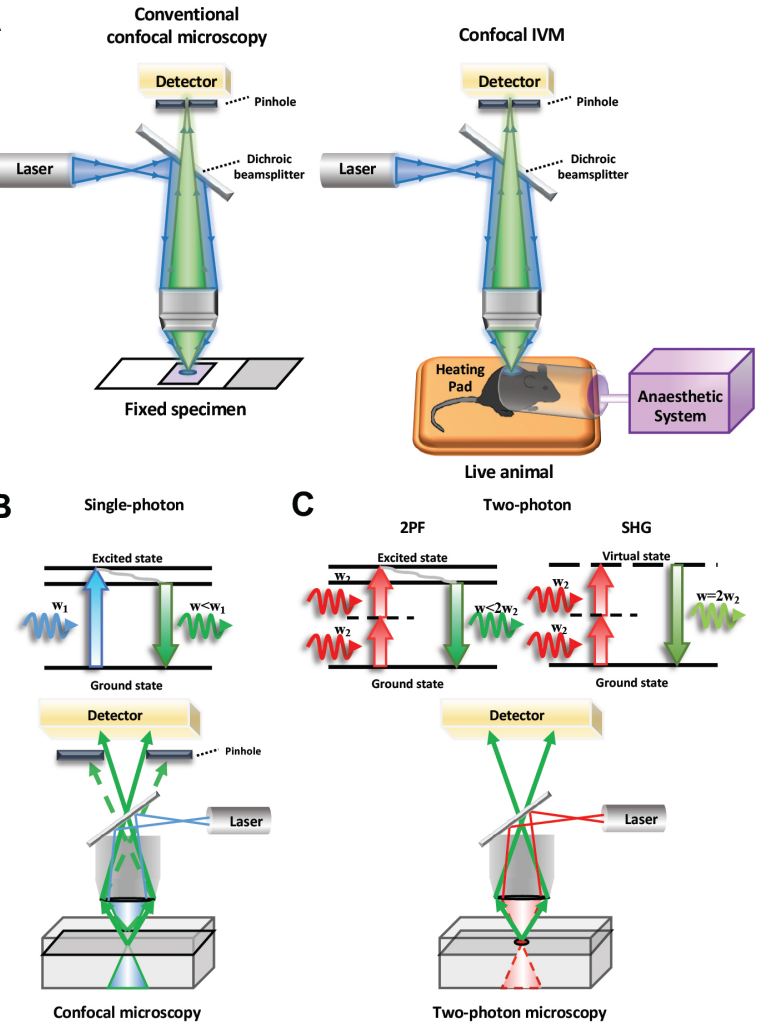

Fig. 1. Schematic of IVM and basic optics of confocal/two-photon microscopy. (A) Comparison of conventional confocal microscopy and confocal IVM. The optics of the two imaging systems are not significantly different. However, whereas a conventional confocal microscope can visualize fixed tissue sections or organs extracted from an animal, confocal IVM allows the obtaining of images from the tissue of a live animal. Therefore, IVM can be equipped with additional devices, such as a heating pad or anaesthetic system, in order to make sure that living objects can breathe comfortably for undisrupted imaging. Schematics of the optics of confocal (B) and two-photon microscopy (C). (B) In confocal microscopy, a single photon has enough energy to excite the sample. The emitted photon has slightly lower energy and frequency $(\omega)$ than the original photon (frequency $\left.\omega_{1}\right)$. In two-photon microscopy, the summed energy of the two photons (frequency $\omega_{2}$ ) is enough to excite the sample to emit fluorescence. The emitted photon has slightly lower energy and frequency $(\omega)$ than the sum of the two photons (frequency $\left.2 \omega_{2}\right)$. Also, with two-photon microscopy, second-harmonic generation (SHG) can be observed when two photons with the same wavelength interact with a non-linear material and then generate a new photon with twice as much energy as the initial photons. The virtual state is not the energy level of the atom, but rather represents the combined energy of photons. Meanwhile, unlike a single photon, a two-photon microscope does not require a pinhole to exclude out-of-focus background signals. Since two-photon excitation generates fluorescence only at the focal plane, there is no background signal. 2PF, two-photon fluorescence; SHG, second-harmonic generation.

properties, multi-photon microscopy is adapted to intravital imaging and used for discovering novel aspects of biology. 


\section{Applications of IVM in various fields of biological and biomedical sciences}

IVM has enabled researchers to observe a variety of biological constructs in live animals and to follow the dynamic behavior of single cells over a long period of time. With these properties, researchers have used IVM for visualizing and analyzing biological processes in various research fields, such as vascular biology, immunology, stem cell biology, and oncology.

In the field of cancer immunology, for instance, Jung et al. used video-rate scanning IVM to discover dynamic tumor infiltration of Ly6C $\mathrm{C}^{\mathrm{lo}}$ non-classical monocytes in the setting of resistance to anti-angiogenic therapy in colorectal cancer (1). In this study, Cx3cr $1^{\mathrm{gfp} /+}$ knock-in mice were used to track motility of the monocytes, and TRITC-dextran was intravenously injected to make the tumor vasculatures visible. Also, a newly developed abdominal imaging window was implanted for longitudinal monitoring of tumors and immune microenvironments (1). The type of motility was defined by the speed of a cell in the study, and the number of flowing (at a speed of $>0.2 \mathrm{~mm} / \mathrm{s})$, rolling $(15-50 \mu \mathrm{m} / \mathrm{s})$, and crawling $(<15 \mu \mathrm{m} / \mathrm{s})$ cells in the blood vessels were measured with this highly advanced in vivo imaging system (1). This study used IVM to see the active tissue infiltration of $\mathrm{Ly}^{6} \mathrm{C}^{\mathrm{lo}}$ non-classical monocytes, which had not been observed or reported in any context using conventional experimental methods. This was the very first direct view of Ly6C ${ }^{\text {lo }}$ non-classical monocytes infiltrating into tissues, especially in the context of anti-angiogenic therapy in tumors $(1,4)$.

IVM has been also used for quantitative evaluation of the organization, structure, and function of blood vasculature. Especially, IVM was used to view irregular hyperpermeability and heterogeneous blood flow in tumor microvessels, resulting from an imbalance of pro- and anti-angiogenic factors. These abnormalities of tumor blood vessels hinder the efficacy of radio-/chemotherapy and promote the growth of more aggressive and metastatic cancer cells (22). Early examination of tumor vessels with fluorescent agents and IVM revealed the difference in static values, such as vessel diameter, length, surface area volume, and branching patterns, between the normal and tumor vessels $(23,24)$. With the development of IVM, dynamic parameters, such as blood flow velocity, changing vessel diameter, and vascular permeability, are quantified and analyzed for a better understanding and classification of blood vessels (25-27). Microscopic observation of these characteristics of vessels in a living animal with IVM can be used to analyze the restoration of vessel functionality and structure after vessel normalization (28). Also, the development of various types of imaging windows permits the longitudinal monitoring of vasculature development over a long period of time (29-34).

Cell trafficking with IVM reveals novel aspects of cellular immunity by its ability to follow and observe the dynamic behavior of immune cells. The high spatial and temporal resolution of IVM enables researchers to track the movement and behavior of immune cells in desired sites (35). Cell-trafficking parameters, such as the population, migration, clustering, 2D/3D movement pattern, velocity, and morphology of immune cells, can be used to understand the states and functions of leukocytes (36). For example, immature T and B lymphocytes migrate to the secondary lymphoid organs and become immunocompetent cells in these sites (37). For detailed comprehension of mechanisms in those processes, early pioneer researchers produced fluorescence-labeled T and B lymphocytes and transferred them to recipient animals to observe their dynamic behavior, such as velocity, moving pattern, $\mathrm{T}$ cell interaction with dendritic cells, morphology changes, and activation in the lymph node (38-40). These studies visualized the dynamic behavior of immune cells and deepened the understanding of adaptive and innate immunity (41). Also, researchers analyzed and quantitatively evaluated the motility of immune cells in the specific condition with IVM. Some studies showed the change in the motility of immune cells by specific molecules. For example, researchers used IVM to reveal how integrins LFA-1 and MAC-1 regulate neutrophil extravasation in the endothelial basement membrane and pericyte sheath (42), and how reactive oxygen species recruit neutrophils to muscle tissue after exercise (43). In addition, IVM was used to show that chemokine CCL22 expression of dendritic cells affects the contact and interaction of dendritic cells with Tregs in the lymph nodes (44). Other studies showed that specific states affect the dynamic patterns and velocity of immune cells. For example, the population and velocity of eosinophils in different organs and their changes in the normal and ovalbumin sensitization state can be seen with IVM (45). Also, IVM was applied to images to analyze how HIV infection changes T-cell motility and migration patterns by disrupting host-cell cytoskeletons (46) and how pathogen-specific T cells interact with intracellular parasite-infected cells (47). In addition, in vivo imaging of tissues with IVM discovered the novel tissue-specific immune responses, including the studies that viewed glycoengineered antibodies potentiate the B-cell depletion by macrophages in the liver (48) and supported other discoveries on liver immunology in various diseases states with IVM (49).

IVM has been also used for viewing the movement and location of stem cells to understand their functions. Some studies used IVM to view hematopoietic stem cells in the bone marrow niche and analyzed their interaction, which is crucial for proper stem-cell functions. For example, some researchers developed IVM-guided transplantation of a hematopoietic stem cell in the bone marrow of mice to study the functional characteristics of a single stem cell (50). Other researchers used IVM to examine the relationship of hematopoietic stem cells and progenitor cells to blood vessels, osteoblasts, and the endosteal surface in the niche $(51,52)$, and others used IVM to view the dynamic responses of the hematopoietic stem cells in the niche upon diverse stresses (53). Also, researchers used IVM to discover novel facts of stem cell function. For example, 
IVM was used to view the interaction of the hematopoietic stem cells with Tregs, which results in Treg accumulation in the niche that prevents immune attacks (54). In another study, IVM was used to analyze the function of mammary stem cells, which regulates the duct-branching morphogenesis (55).

In the field of oncology, IVM is applied to view the singlecell behavior of cancer and immune cells during the progression and metastasis of the tumor, which can reveal new findings in the tumor microenvironment. Imaging the singlecell behavior enables researchers to understand the relationships between the dynamic behavior of cancer cells and the features of the tumor (56). Also, cancer-cell invasion and metastasis, which are the primary reason for the death of cancer patients (57), are made visible and explored by IVM. For instance, IVM was used to view circulating tumor cells, which are known to be highly associated with metastasis and defined their higher metastatic subsets (58). Also, some studies observe the pre-metastasis stage in a liver metastasis model with an abdominal imaging window (59). Another study developed an intravital imaging model of cancer cells in bone marrow to study systemic metastasis (60). In addition, some researchers have used IVM to view and analyze the interaction of cancer cells with immune cells. IVM is used in studies that imaged and analyzed the interaction between tumor-infiltrating $T$ lymphocytes with cancer cells (61) and how adopted cytotoxic CD8 + T lymphocytes induce tumor-cell death (62). Also, other researchers used IVM to reveal the mechanism of how tumorassociated macrophages are related to poor prognosis by viewing macrophage-assisted cancer-cell intravasation (63) and tumor-associated macrophage-mediated drug resistance (64). The increasing number of cancer studies now use IVM to discover and render unexpected findings in the tumor microenvironment.

As mentioned above, IVM has a limited penetration depth, which is an obstacle in imaging deep tissues, such as the brain, colon, and heart (65). Endomicroscopy based on the optical probe is a method that overcomes this obstacle and can observe the deep organs. Micro-diameter probes using graded refractive index (GRIN) lenses enable conventional intravital microscopes to view the internal deep organs of animals with minimal invasion. Some organs can even be viewed without incision or additional components. For example, colon vessels and tumorigenesis are observed by endomicroscopy that enters through the rectum without

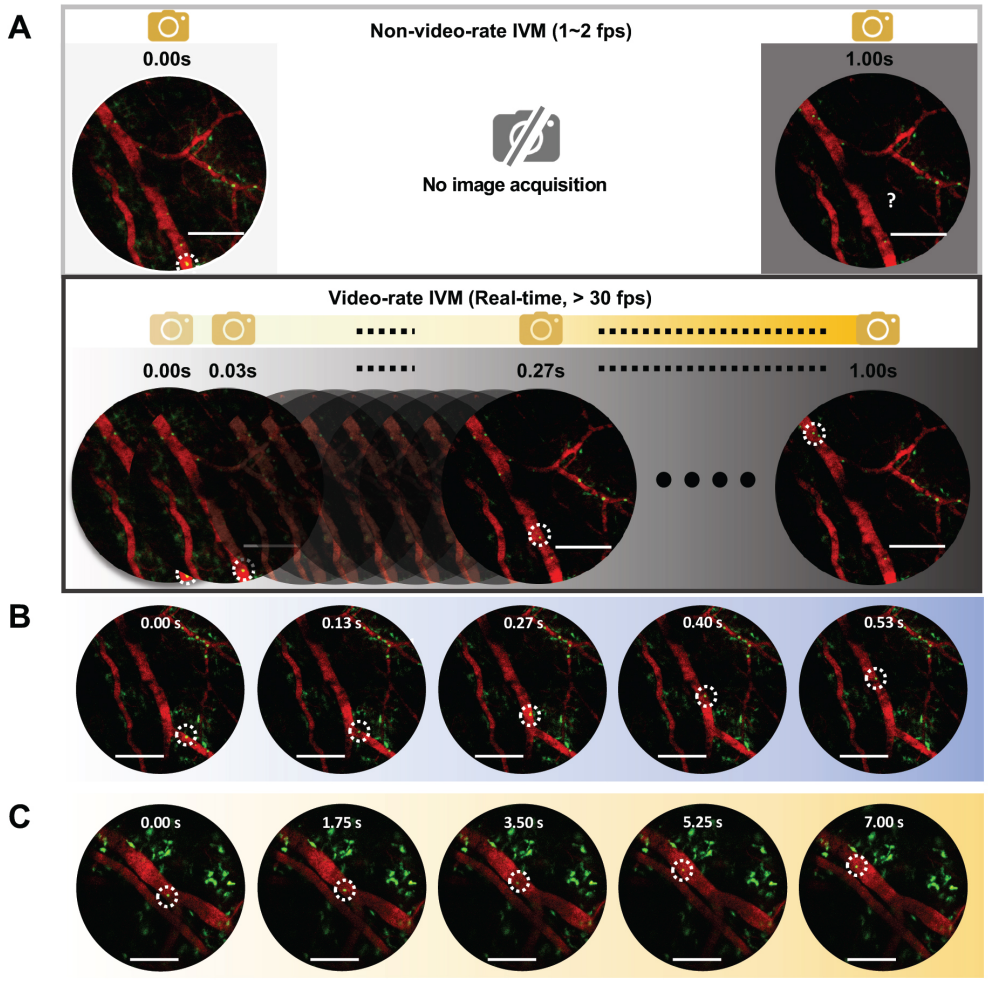

Fig. 2. Comparison of conventional and real-time IVM. (A) Comparison of the frame rates of conventional IVM (non-video-rate, 1-2 fps) and real-time IVM (video-rate, $30 \mathrm{fps}$ ). Ear vessel of CX3CR1-GFP mice is shown. The white dotted ring indicates a flowing cell. Yellow camera icons indicate the timepoints for taking images. The flowing cell (B) and rolling cell (C) are tracked by real-time IVM. Ear vessel of CX3CR1-GFP mice is shown. The white dotted ring indicates the moving cells. Scale bar: $100 \mu \mathrm{m}$. RED, RITC-dextran 70. 
invasion $(35,66,67)$. Also, mesenchymal stem-cell therapy in the bladder-pain syndrome model can be analyzed by endomicroscopy that enters the bladder without an incision (68). On the other hand, viewing the brain with endomicroscopy needs a small incision to implant the probe. To minimize the damage and inflammation, some researchers fabricated a side-view probe with the small GRIN rod lenses with a micro-diameter and viewed the murine brain from the cortex to the hypothalamus (69) and the hippocampus (70). With similar methods, intravital endomicroscopy can make the glioma progression in the brain visible (71). In addition, viewing the heart or airway needs additional caution, because of the vigorous movement of the heartbeat and breathing. To view the heart, researchers used a suction tube to locally stabilize the movement of the heart and examined the flowing and rolling monocytes in the beating heart (2). In airway imaging, the challenge by breathing was overcome by the side-view design, which results in proximity between the epithelium of the airway and the imaging window of the probe (72).

With the development of IVM and endomicroscopy, the clinical adaptation of IVM starts in various studies (16). Fluorescein is the most widely used in the clinical adaptation of IVM, because fluorescein is FDA-approved for angiography of the retina (73). Diagnostic criteria for benign and neoplastic or tumor conditions has been developed by probe-based IVM and fluorescein in the colon (74), esophagus (75), and urinary tract (76). Also, one study emphasizes another important aspect of intravital imaging, as IVM-imaged human tumor vessel diameters in melanoma patients are larger than predicted from immunohistochemistry. Thus, the measurement of vessel diameters with IVM enables to establish more precise strategies for drug delivery and immunotherapies (77).

\section{Challenge in conventional IVM: The importance of real-time IVM}

Although conventional IVM has proved to be an excellent tool for in vivo imaging, the limited frame rate (1-2 frames/sec, in general) of most of the early IVM was an obstacle for observing the rapid dynamic behavior of cells (78). To overcome this obstacle, real-time IVM that is capable of video-rate image scanning (higher than 30 frames per second) was invented to visualize and analyze those faster movements of cells and to discover new aspects of the function and interaction of cells (78).

Fig. 2 compares conventional (non-video-rate scanning) and real-time (video-rate scanning) IVM. The limited frame rate of conventional IVM cannot track the movement of flowing cells. On the other hand, the rapid movement of the flowing cell can be tracked with real-time IVM (Fig. 2A). Also, the different movements of cells such as flowing (Fig. 2B) and rolling (Fig. $2 \mathrm{C}$ ), can be analyzed by real-time IVM, which is related to their function and population at the site of imaging. This high-temporal resolution of real-time IVM expands the visual perception of researchers for understanding the dynamic cel- lular behavior, which leads to novel discovery.

Fig. 3 is a schematic of real-time confocal and two-photon switchable IVM with two different options for beam scanner systems. In the upper panel, a schematic shows an example of real-time IVM that has two different modes of intravital imaging. The backscattered light or fluorescent light from the tissue of a living animal was collected by the objective lens and delivered to either one of the two independent PMTs, depending on the mode of imaging (79). A photomultiplier tube with a confocal pinhole is built to detect confocal fluorescence and reflectance imaging. Another one (two-photon PMT) is built to detect two-photon fluorescence and second-

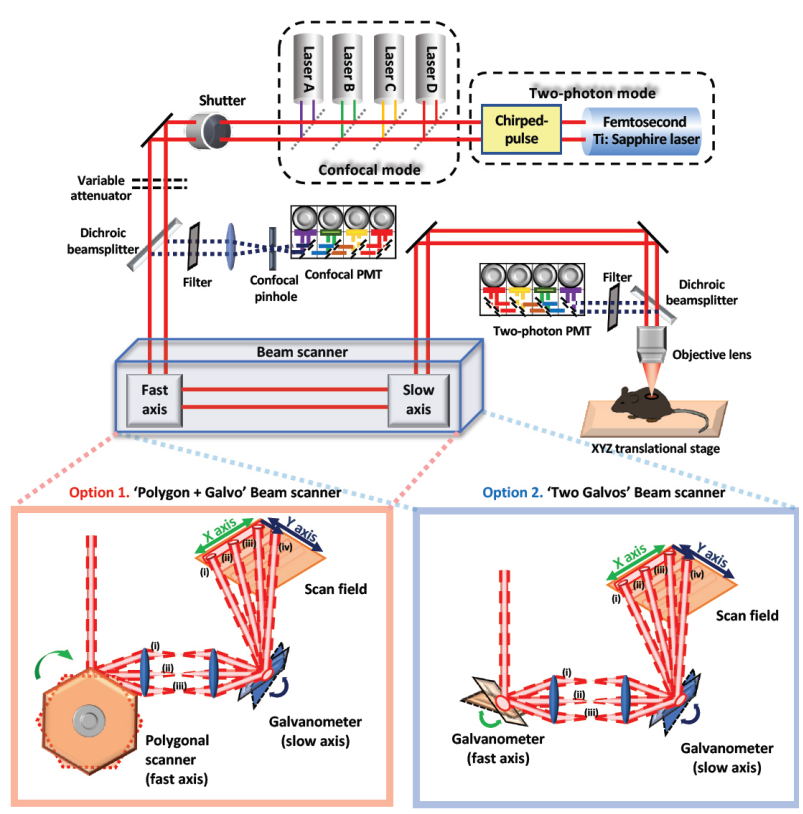

Fig. 3. The schematic of real-time confocal and two-photon switchable intravital microscopy. (Upper panel) A schematic of the real-time confocal and two-photon intravital microscopy. In confocal mode, the continuous lasers excite the sample, and the emitted light is detected by confocal PMT. In two-photon mode, a Ti:Sapphire (femtosecond) laser shots red and near-infrared light, and the laser goes through a chirped-pulse amplifier, a special component that is necessary to prevent the pulse from damaging the parts in the laser. Then light excites the focal region of the sample. The twophoton PMT, which is closer to the objective lens, detects the two-photon fluorescence and second-harmonic generation signal. (Lower panel) The beam scanner can be operated via two different systems. Option 1 is a beam scanner consisting of a polygonal scanner (for the fast axis) and a galvanometer (for the slow axis). Option 2 is a beam laser consisting of two galvanometers (one for the fast axis, the other for the slow axis). Two different combinations can be used in real-time IVM for higher frame rates. The light path moves from (i) through (ii) to (iii), guided by the fast axis. The guided light then scans an x-axis line on the scan field. The light path then moves to (iv) along the $y$-axis, directed by the slow axis. Next, the light scans a next x-axis line on the scan field. Galvo, galvanometer; PMT, photomultiplier tube. 


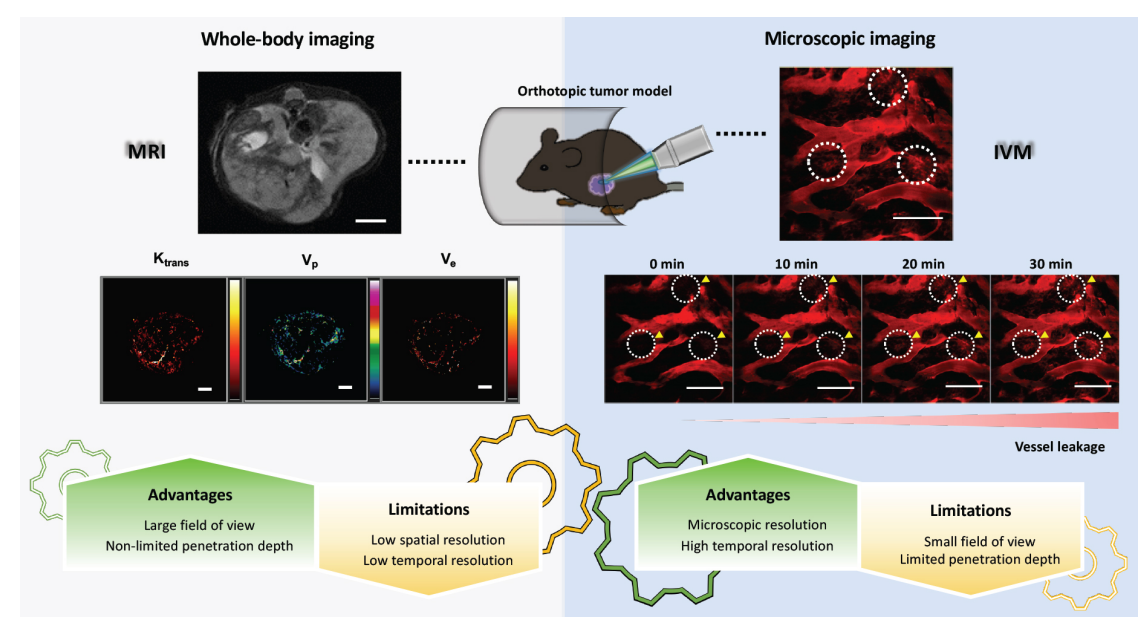

Fig. 4. Complementary relationship between whole-body imaging and IVM. Complementary relationship between MRI (whole-body imaging) and IVM. Tumor vessel leakage in the colon-cancer origin liver-metastasis model is evaluated by MRI and IVM. The combined use of whole-body imaging and IVM can complement the weakness of each imaging system and can generate synergistic effects. The white dotted rings and yellow triangles indicate increasing vessel leakage over time. Scale bar in MRI, $5 \mathrm{~mm}$. Scale bar in IVM, $100 \mu \mathrm{m}$. RED in IVM data, RITC-dextran. K Ktrans, transfer constant from the blood plasma into the extracellular extravascular space; $V_{p}$, fractional blood plasma volume; $V_{e}$, extracellular extravascular volume fraction.

harmonic generation imaging. In confocal microscopy mode, the emitted light from the tissue of the animal returns along the same path as the excitation laser until it is reflected at the dichroic beamsplitter, then the confocal pinhole selectively transmits only fluorescence generated at the focal region. In contrast, because the fluorescence originates only at a point on the focal region in two-photon microscopy mode, it does not require pinhole for blocking background signal. Thus, the emitted fluorescence or SHG signals can be sent directly to an optical dector, which can be placed as close to the tissue of the animal as possible to minimize the loss of the emitted light (80). In the lower panel, the scanning by two different scanners is described. The frame rate of IVM is determined by the speed of vertical and horizontal scan mirrors that move the laser across the $x-y$ plane of the scan field. To achieve a higher frame rate, researchers used a galvanometer and high-speed rotating polygonal scanner as "Option 1" $(78,79,81)$, or horizontal linear scanning mirror with an oscillating resonant scanning mirror as "Option 2" (82). When the fast axis rotates (polygonal scanner) or oscillates (galvanometer mirror), the light path moves from (i) through (ii) to (iii) in $\mathrm{x}$-axis line and line scanning occurs along the $x$-axis of a scan field. Then, the slow axis tilts the light path to move along the $y$-axis (iv), and the fast axis moves again, and scanning goes on.

The researchers used real-time IVM to view swift biological processes, such as the significant motion of tracheal epithelial cells caused by breathing and heartbeat (72), tumor development in gastrointestinal tracts (83), and the rapid movements of immune cells in cancers (1) and cardiovascular system (2). In addition, this real-time IVM can analyze and evaluate the delivery and effects of nanotherapeutics in living animals (84,
85). In short, real-time IVM will serve as a sophisticated tool for visualizing rapid biological events, which cannot be achieved by non-video-rate scanning IVM.

\section{CONCLUSION}

IVM incorporates diverse optical systems for direct viewing and analyzing the biological structure and dynamic behavior of cells in living animals. Unlike ex vivo analyses or wholebody imaging, IVM can show the biological process in a living organism in higher spatial and temporal resolution. Therefore, IVM has positively influenced the breakthrough of novel findings in various fields of vascular biology, immunology, stem cell biology, oncology, and so on. Furthermore, real-time IVM will enable us to observe and analyze cellular dynamics for a better understanding of the complexity of biological system (1, $2,4,41,86)$.

Nontheless, compared to other in vivo imaging modalities, IVM has relatively small field of view and limited penetration depth, which can be a hindrance to diverse IVM applications. Also, the necessity of fluorescence would be an obstacle to clinical applications.

On the other hand, whole-body imaging systems, which has been widely used in the clinic, are suitable for viewing large parts of tissues and analyzing the function of whole tissues, although these methods in general have low spatial resolution, unlike IVM. Thus, the use of IVM and whole-body imaging systems can be well combined to obtain more comprehensive biological information that cannot be obtained by a single method (Fig. 4). IVM and whole-body imaging modalities compensate for the weakness of each other's imaging system. 
Also, the constant development of IVM will surely broaden our knowledges on the field of biological and biomedical sciences by providing novel insights, which cannot be achieved by conventional experimental techniques.

\section{ACKNOWLEDGEMENTS}

This study was supported by the Creative-Pioneering Researchers Program through Seoul National University (SNU) (KJ) and by the National Research Foundation of Korea (NRF) grant funded by the Korea government (MSIT) (No. 2020R1C1C10 15062), by the Cooperative Research Program of Basic Medical Science and Clinical Science from the Seoul National University College of Medicine (800-20190261) (KJ), by grant no. 162019-007 from the Seoul National University Bundang Hospital research fund $(\mathrm{KJ})$, and by the SNUH Research Fund 032018-0290 (KJ).

\section{CONFLICTS OF INTEREST}

The authors have no conflicting interests.

\section{REFERENCES}

1. Jung K, Heishi T, Khan OF et al (2017) Ly6Clo monocytes drive immunosuppression and confer resistance to antiVEGFR2 cancer therapy. J Clin Invest 127, 3039-3051

2. Jung K, Kim P, Leuschner F et al (2013) Endoscopic timelapse imaging of immune cells in infarcted mouse hearts. Circ Res 112, 891-899

3. Choi M, Kwok SJ and Yun SH (2015) In vivo fluorescence microscopy: lessons from observing cell behavior in their native environment. Physiology (Bethesda) 30, 40-49

4. Jung K, Heishi T, Incio J et al (2017) Targeting CXCR4dependent immunosuppressive Ly6C(low) monocytes improves antiangiogenic therapy in colorectal cancer. Proc Natl Acad Sci U S A 114, 10455-10460

5. Weigert R, Sramkova M, Parente $L$, Amornphimoltham $P$ and Masedunskas A (2010) Intravital microscopy: a novel tool to study cell biology in living animals. Histochem Cell Biol 133, 481-491

6. Leblond F, Davis SC, Valdés PA and Pogue BW (2010) Pre-clinical whole-body fluorescence imaging: Review of instruments, methods and applications. J Photochem Photobiol B 98, 77-94

7. Condeelis J and Weissleder R (2010) In vivo imaging in cancer. Cold Spring Harb Perspect Biol 2, a003848

8. Cahalan MD and Parker I (2008) Choreography of cell motility and interaction dynamics imaged by two-photon microscopy in lymphoid organs. Annu Rev Immunol 26, 585-626

9. Sanderson MJ, Smith I, Parker I and Bootman MD (2014) Fluorescence microscopy. Cold Spring Harb Protoc 2014, pdb top071795

10. Greenberg ML, Weinger JG, Matheu MP et al (2014) Two-photon imaging of remyelination of spinal cord axons by engrafted neural precursor cells in a viral model of multiple sclerosis. Proc Natl Acad Sci U S A 111, E23492355

11. Entenberg D, Wyckoff J, Gligorijevic B et al (2011) Setup and use of a two-laser multiphoton microscope for multichannel intravital fluorescence imaging. Nat Protoc 6, 15001520

12. Hierro-Bujalance C, Bacskai BJ and Garcia-Alloza M (2018) In vivo imaging of microglia with multiphoton microscopy. Front Aging Neurosci 10, 218

13. Lyons SK (2005) Advances in imaging mouse tumour models in vivo. J Pathol 205, 194-205

14. Andresen V, Pollok K, Rinnenthal JL et al (2012) Highresolution intravital microscopy. PLoS One 7, e50915

15. Ivashchenko $O$, van der Have F, Villena JL et al (2015) Quarter-millimeter-resolution molecular mouse imaging with U-SPECT(+). Mol Imaging 14, 7290.2014.00053

16. Gabriel EM, Fisher DT, Evans S, Takabe K and Skitzki JJ (2018) Intravital microscopy in the study of the tumor microenvironment: from bench to human application. Oncotarget 9, 20165-20178

17. Lu FM and Yuan Z (2015) PET/SPECT molecular imaging in clinical neuroscience: recent advances in the investigation of CNS diseases. Quant Imaging Med Surg 5, 433-447

18. Wang W, Wyckoff JB, Frohlich VC et al (2002) Single cell behavior in metastatic primary mammary tumors correlated with gene expression patterns revealed by molecular profiling. Cancer Res 62, 6278

19. Norman K (2005) Techniques: intravital microscopy-a method for investigating disseminated intravascular coagulation? Trends Pharmacol Sci 26, 327-332

20. Gavins FNE and Chatterjee BE (2004) Intravital microscopy for the study of mouse microcirculation in antiinflammatory drug research: focus on the mesentery and cremaster preparations. J Pharmacol Toxicol Methods 49, $1-14$

21. Pantazis P, Maloney J, Wu D and Fraser SE (2010) Second harmonic generating (SHG) nanoprobes for in vivo imaging. Proc Natl Acad Sci U S A 107, 14535-14540

22. Fukumura D, Duda DG, Munn LL and Jain RK (2010) Tumor microvasculature and microenvironment: novel insights through intravital imaging in pre-clinical models. Microcirculation 17, 206-225

23. Yuan F, Chen Y, Dellian M, Safabakhsh N, Ferrara N and Jain RK (1996) Time-dependent vascular regression and permeability changes in established human tumor xenografts induced by an anti-vascular endothelial growth factor/ vascular permeability factor antibody. Proc Natl Acad Sci U S A 93, 14765-14770

24. Yuan F, Salehi HA, Boucher Y, Vasthare US, Tuma RF and Jain RK (1994) Vascular permeability and microcirculation of gliomas and mammary carcinomas transplanted in rat and mouse cranial windows. Cancer Res 54, 4564-4568

25. Honkura N, Richards M, Lavina B, Sainz-Jaspeado M, Betsholtz C and Claesson-Welsh L (2018) Intravital imaging-based analysis tools for vessel identification and assessment of concurrent dynamic vascular events. Nat Commun 9, 2746

26. Jain RK, Munn LL and Fukumura D (2002) Dissecting tumour pathophysiology using intravital microscopy. Nat Rev Cancer 2, 266-276 
27. Meijer EFJ, Blatter C, Chen IX et al (2017) Lymph node effective vascular permeability and chemotherapy uptake. Microcirculation 24, e12381

28. Kirkpatrick ND, Chung E, Cook DC et al (2012) Video-rate resonant scanning multiphoton microscopy: an emerging technique for intravital imaging of the tumor microenvironment. IntraVital 1, 60-68

29. Askoxylakis V, Badeaux M, Roberge $S$ et al (2017) A cerebellar window for intravital imaging of normal and disease states in mice. Nat Protoc 12, 2251-2262

30. Meijer EFJ, Jeong HS, Pereira ER et al (2017) Murine chronic lymph node window for longitudinal intravital lymph node imaging. Nat Protoc 12, 1513-1520

31. Ellenbroek SI and van Rheenen J (2014) Imaging hallmarks of cancer in living mice. Nat Rev Cancer 14, 406418

32. Alieva M, Ritsma L, Giedt RJ, Weissleder R and van Rheenen J (2014) Imaging windows for long-term intravital imaging: general overview and technical insights. Intravital 3, e29917

33. Entenberg D, Voiculescu S, Guo P et al (2018) A permanent window for the murine lung enables highresolution imaging of cancer metastasis. Nat Methods 15, $73-80$

34. Entenberg D, Rodriguez-Tirado C, Kato Y, Kitamura T, Pollard JW and Condeelis J (2015) In vivo subcellular resolution optical imaging in the lung reveals early metastatic proliferation and motility. IntraVital 4, 1-11

35. Kim JK, Lee WM, Kim P et al (2012) Fabrication and operation of GRIN probes for in vivo fluorescence cellular imaging of internal organs in small animals. Nat Protoc 7, 1456-1469

36. Matheu MP, Cahalan MD and Parker I (2014) Intravital Imaging of the Immune System; in Advances in Intravital Microscopy: From Basic to Clinical Research, Weigert $\mathrm{R}$ (ed.), 81-103, Springer Netherlands, Dordrecht

37. Nitschke C, Garin A, Kosco-Vilbois $M$ and Gunzer M (2008) 3D and 4D imaging of immune cells in vitro and in vivo. Histochem Cell Biol 130, 1053-1062

38. Mempel TR, Henrickson SE and von Andrian UH (2004) T-cell priming by dendritic cells in lymph nodes occurs in three distinct phases. Nature 427, 154-159

39. Miller MJ, Safrina O, Parker I and Cahalan MD (2004) Imaging the single cell dynamics of CD4 + T cell activation by dendritic cells in lymph nodes. J Exp Med 200, 847-856

40. Miller MJ, Wei SH, Parker I and Cahalan MD (2002) Two-photon imaging of lymphocyte motility and antigen response in intact lymph node. Science 296, 1869-1873

41. Germain RN, Robey EA and Cahalan MD (2012) A decade of imaging cellular motility and interaction dynamics in the immune system. Science 336, 1676-1681

42. Hyun YM, Choe YH, Park SA and Kim M (2019) LFA-1 (CD11a/CD18) and Mac-1 (CD11b/CD18) distinctly regulate neutrophil extravasation through hotspots I and II. Exp Mol Med 51, 1-13

43. Nunes-Silva A, Bernardes PT, Rezende BM et al (2014) Treadmill exercise induces neutrophil recruitment into muscle tissue in a reactive oxygen species-dependent manner. An intravital microscopy study. PLoS One 9, e96464
44. Rapp M, Wintergerst MWM, Kunz WG et al (2019) CCL22 controls immunity by promoting regulatory $\mathrm{T}$ cell communication with dendritic cells in lymph nodes. J Exp Med 216, 1170-1181

45. Chojnacki A, Wojcik K, Petri B et al (2019) Intravital imaging allows real-time characterization of tissue resident eosinophils. Commun Biol 2, 181

46. Usmani SM, Murooka TT, Deruaz M et al (2019) HIV-1 balances the fitness costs and benefits of disrupting the host cell actin cytoskeleton early after mucosal transmission. Cell Host Microbe 25, 73-86. e5

47. Filipe-Santos O, Pescher P, Breart B et al (2009) A dynamic map of antigen recognition by CD4 T cells at the site of Leishmania major infection. Cell Host Microbe 6, 2333

48. Grandjean CL, Montalvao F, Celli S et al (2016) Intravital imaging reveals improved Kupffer cell-mediated phagocytosis as a mode of action of glycoengineered anti-CD20 antibodies. Sci Rep 6, 34382

49. Marques PE, Oliveira AG, Chang L, Paula-Neto HA and Menezes GB (2015) Understanding liver immunology using intravital microscopy. J Hepatol 63, 733-742

50. Turcotte R, Alt C, Runnels JM et al (2017) Image-guided transplantation of single cells in the bone marrow of live animals. Sci Rep 7, 3875

51. Lo Celso C, Fleming HE, Wu JW et al (2009) Live-animal tracking of individual haematopoietic stem/progenitor cells in their niche. Nature 457, 92-96

52. Khorshed RA, Hawkins ED, Duarte D et al (2015) Automated identification and localization of hematopoietic stem cells in 3d intravital microscopy data. Stem Cell Rep 5, 139-153

53. Batsivari A, Haltalli MLR, Passaro D, Pospori C, Lo Celso C and Bonnet D (2020) Dynamic responses of the haematopoietic stem cell niche to diverse stresses. Nat Cell Biol 22, 7-17

54. Fujisaki J, Wu J, Carlson AL et al (2011) In vivo imaging of Treg cells providing immune privilege to the haematopoietic stem-cell niche. Nature 474, 216-219

55. Scheele $C L$, Hannezo E, Muraro MJ et al (2017) Identity and dynamics of mammary stem cells during branching morphogenesis. Nature 542, 313-317

56. Pinner S, Jordan P, Sharrock K et al (2009) Intravital imaging reveals transient changes in pigment production and Brn2 expression during metastatic melanoma dissemination. Cancer Res 69, 7969-7977

57. Chaffer $C$ and Weinberg R (2011) A perspective on cancer cell metastasis. Science 331, 1559-1564

58. Kuo CW, Chueh DY and Chen P (2019) Real-time in vivo imaging of subpopulations of circulating tumor cells using antibody conjugated quantum dots. J Nanobiotechnology 17,26

59. Ritsma L, Steller EJA, Beerling E et al (2012) Intravital microscopy through an abdominal imaging window reveals a pre-micrometastasis stage during liver metastasis. Sci Transl Med 4, 158ra145

60. Lee SH, Park SA, Zou Y et al (2018) Real-time monitoring of cancer cells in live mouse bone marrow. Front Immunol 9, 1681

61. Ng LG, Mrass P, Kinjyo I, Reiner SL and Weninger W 
(2008) Two-photon imaging of effector T-cell behavior: lessons from a tumor model. Immunol Rev 221, 147-162

62. Breart B, Lemaitre F, Celli S and Bousso P (2008) Twophoton imaging of intratumoral CD8 $+\mathrm{T}$ cell cytotoxic activity during adoptive $\mathrm{T}$ cell therapy in mice. J Clin Invest 118, 1390-1397

63. Wyckoff JB, Wang Y, Lin EY et al (2007) Direct visualization of macrophage-assisted tumor cell intravasation in mammary tumors. Cancer Res 67, 2649-2656

64. Arlauckas SP, Garris CS, Kohler RH et al (2017) In vivo imaging reveals a tumor-associated macrophage-mediated resistance pathway in anti-PD-1 therapy. Sci Transl Med 9, eaal3604

65. Barretto RP and Schnitzer MJ (2012) In vivo optical microendoscopy for imaging cells lying deep within live tissue. Cold Spring Harb Protoc 2012, 1029-1034

66. Paulson B, Kim IH, Namgoong JM et al (2019) Longitudinal micro-endoscopic monitoring of high-success intramucosal xenografts for mouse models of colorectal cancer. Int J Med Sci 16, 1453-1460

67. Choi JW, Kim JK, Choi M, Kim YR and Yun SH (2014) In vivo imaging of Lgr5-positive cell populations using confocal laser endomicroscopy during early colon tumorigenesis. Endoscopy 46, 1110-1116

68. Kim A, Yu HY, Lim J et al (2017) Improved efficacy and in vivo cellular properties of human embryonic stem cell derivative in a preclinical model of bladder pain syndrome. Sci Rep 7, 8872

69. Kim JK, Choi JW and Yun SH (2013) 350-mum side-view optical probe for imaging the murine brain in vivo from the cortex to the hypothalamus. J Biomed Opt 18, 50502

70. Barretto RP and Schnitzer MJ (2012) In vivo microendoscopy of the hippocampus. Cold Spring Harb Protoc 2012, 1092-1099

71. Barretto RP, Ko TH, Jung JC et al (2011) Time-lapse imaging of disease progression in deep brain areas using fluorescence microendoscopy. Nat Med 17, 223-228

72. Kim JK, Vinarsky V, Wain J et al (2012) In vivo imaging of tracheal epithelial cells in mice during airway regeneration. Am J Respir Cell Mol Biol 47, 864-868

73. Wallace MB, Meining A, Canto Ml et al (2010) The safety of intravenous fluorescein for confocal laser endomicroscopy in the gastrointestinal tract. Aliment Pharmacol Ther $31,548-552$
74. Xie XJ, Li CQ, Zuo XL et al (2011) Differentiation of colonic polyps by confocal laser endomicroscopy. Endoscopy 43, 87-93

75. Kiesslich R, Gossner L, Goetz $M$ et al (2006) In vivo histology of Barrett's esophagus and associated neoplasia by confocal laser endomicroscopy. Clin Gastroenterol Hepatol 4, 979-987

76. Wu K, Liu JJ, Adams W et al (2011) Dynamic real-time microscopy of the urinary tract using confocal laser endomicroscopy. Urology 78, 225-231

77. Fisher DT, Muhitch JB, Kim M et al (2016) Intraoperative intravital microscopy permits the study of human tumour vessels. Nat Commun 7, 10684

78. Padera TP, Stoll BR, So PTC and Jain RK (2002) Conventional and high-speed intravital multiphoton laser scanning microscopy of microvasculature, lymphatics, and leukocyte-endothelial interactions. Mol Imaging 1, 9-13

79. Kim P, Puoris'haag M, Cote D, Lin CP and Yun SH (2008) In vivo confocal and multiphoton microendoscopy. J Biomed Opt 13, 010501

80. Benninger RKP and Piston DW (2013) Two-photon excitation microscopy for the study of living cells and tissues. Curr Protoc Cell Biol Chapter 4, Unit 4.11.1-24

81. Veilleux I, Spencer JA, Biss DP, Cote D and Lin CP (2008) In vivo cell tracking with video rate multimodality laser scanning microscopy. IEEE J Sel Top Quantum Electron $14,10-18$

82. Sanderson MJ (2004) Acquisition of multiple real-time images for laser scanning microscopy. Microsc Anal 18, $17-23$

83. Kim P, Chung E, Yamashita $H$ et al (2010) In vivo wide-area cellular imaging by side-view endomicroscopy. Nat Methods 7, 303-305

84. van de Ven A, Kim P, Ferrari M and Yun S (2013) Real-time intravital microscopy of individual nanoparticle dynamics in liver and tumors of live mice. Protoc Exch 2013, 10.1038/protex.2013.049

85. Kirui DK and Ferrari M (2015) Intravital microscopy imaging approaches for image-guided drug delivery systems. Curr Drug Targets 16, 528-541

86. Jeong J, Suh Y and Jung K (2019) Context Drives Diversification of Monocytes and Neutrophils in Orchestrating the Tumor Microenvironment. Front Immunol 10, 1817 